\title{
A Pilot Study About Computer-Assisted Spine Surgery
}

\author{
L.-P. Nolte ${ }^{1}, H$. Visarius $^{1}$, L. Zamorano ${ }^{2}$ \\ ${ }^{1}$ M.E. Müller Institute for Biomechanics, University of Bern, 3010 Bern, CH \\ ${ }^{2}$ Dept. of Neurological Surgery, Wayne State University, Detroit, MI, USA
}

Introduction. Spinal fixation relying on transpedicular screws was introduced in the early 1960s and has recently been the focus of increased attention. Despite the improved fixation many problems are still involved in the process of screw insertion. Successful placement of a pedicle screw requires the accurate identification of the ideal screw axis. Biomechanical studies suggest an optimal position for the screw tip to be as near to the anterior cortex of the vertebral body as possible. Any major deviation causes perforation of the pedicle or the anterior vertebral cortex and may result in neurological or vascular damage.

Morphometric measurements of the critical pedicle dimensions, i.e. the transverse width and angle have revealed significant variability. Moreover, it has been shown by three-dimensional reconstruction of tomographic images that the greatest width and optimal angle measured from a single image slice may not correspond to the true optimal screw axis through the pedicle. This is confirmed clinically as considerable failure rates in screw location and complications have been reported. We propose a novel technique for spinal surgery that combines preoperative tomographic imaging with principles of stereotaxis. It provides a sound basis for surgical planning and simulation as well as real-time visualization of surgical tools.

Principles of Image Guided Stereotactic Spine Surgery. The proposed system for computer assisted surgery (CAS) is conceptually based on stereotaxis, a method for guided localization of surgical objects within body parts without direct access to their interior. Despite the formulation of the basic principles early this century only recent technology transfer from medical imaging, computer science, and space digitization allowed for an implementation into computer assisted surgery. Systems for frame-based tumor stereotaxis have been reported since the late '70s.

All stereotactic components, the navigator (e.g. spine surgery tools, pointers), the surgical object (e.g. anatomic hard tissue structures such as vertebrae), and the associated virtual object (e.g. medical images) are treated as rigid bodies. Mathematically, the location of a rigid body in space can be determined by the location of three or more non-collinear points (markers) on the body with respect to a global spacefixed coordinate system (COS). The markers are described in a local body-fixed COS. Various techniques exist to track and control a rigid body. In medical applications articulated arms or robots as well as infrared or acoustic motion analysis systems are currently in use.

The following criteria were applied to our stereotactic tracking system. The device should: (a) not interfere with and require only slight modifications of standard surgical procedures and instrumentation, (b) keep surgeons "in the loop", (c) provide sufficient accuracy, and (d) be fast enough to allow real-time instrument control and visualization.

Based on these criteria we selected an opto-electronic motion analysis system, the OPTOTRAK 3020 (Northern Digital, Waterloo, CAN) for intra-operative digitization. Probes instrumented with light emitting diodes (LED) can easily be mounted on existing surgical instruments and/or anatomic structures. Both, the vertebra and the camera may move during the surgical procedure without affecting the accuracy of the system.

Spinal Skeletal Registration. Generally, skeletal registration is required to enable coordinate transformation of the "real world" data of the operative situs into the "virtual world" data of the CT image. For the spine it includes the registration of the bony surface of each vertebra involved in the procedure. In order to obtain a sufficiently accurate transformation within a tolerable time a combination of paired point and surface matching methods were used for this study.

Surgical System and Procedures. The system has been applied in over 200 cases of neurological surgery and three cases (7 vertebrae) of spinal fusion. OSPS - As a basis for the proposed surgical system we use the Orthopaedic Surgery Planning System (OSPS). The modular features of the OSPS software environment have been described in recent papers. Special matching, planning, tracking, and visualization 
algorithms which meet all the outlined requirements of image guided computer assisted spine surgery have been developed and integrated into the OSPS environment.

Pre-operative steps - Tomographic scans, CT and/or MRI, are obtained of all vertebrae to be operated on. A tomographic image visualization module reconstructs and displays the three-dimensional vertebral images in multiple two-dimensional views. An approximate axis for pedicle screw insertion is created by defining its entry and target point by the surgeon. Equidistant serial sections orthogonal to the chosen trajectory (surgeon's perspective) may be displayed to check the insertion axis along the entire pedicle.

Intra-operative steps - Our tool set for image guided pilot hole preparation consists of: (a) a space pointer (Northern Digital, Waterloo, CAN) for digitization of points on the vertebral surface, (b) a standard $A O$ pneumatic hand drill (Synthes, Waldenburg, $\mathrm{CH}$ ) with a $3.6 \mathrm{~mm}$ drill bit, (c) a custom opto-electronic device for intra-operative calibration of the drill, and (d) a custom vertebral marker carrier.

The vertebral marker carrier must be fixed to the vertebra, so that there is no relative motion between the vertebra and this carrier. A suitable and accessible anchorage point is normally provided by the spinous process. For the paired point skeletal registration the pre-operatively chosen anatomic landmarks are located and digitized with the space pointer. To check the quality of the obtained transformation the OSPS position tracking module offers the automatic real-time display of tomographic sections corresponding to any point on the vertebra digitized with the space pointer. In case of insufficient accuracy the surface matching can be applied. For this procedure thirty to sixty points on the accessible vertebral surface are digitized. Once the matching process is completed the surgeon precisely locates and marks the pre-operatively defined entry point with the space pointer.

Before preparation of the pilot hole the pneumatic drill is calibrated which is done hand-hold. The calibrated drill acts as a tracking device initiating the real-time display of the tomographic sections corresponding to the tip of the bit. During the actual drilling phase guiding functions support the surgeon to match the chosen trajectory: real-time display of (a) colored lines in the sagittal, transverse, and frontal images representing the drill's bit, (b) a circle matching the screw diameter displayed in the actual slice of the surgeon's perspective, (c) distances between the tip of the drill's bit and the target point as well as between the tip of the drill's bit and the trajectory, and (d) angle between the axis of the drill's bit and the trajectory.
In Vitro Application. Two human lumbar spines were removed within 24 hours of death. CT scans were obtained of both spines (SIEMENS Somatom Plus-S, Erlangen, FRG) with $2 \mathrm{~mm}$ thick slices spaced $1 \mathrm{~mm}$ and $2 \mathrm{~mm}$ apart. After disarticulation each single vertebra was fixed to a reference frame with the spinous process pointing upwards imitating the intraoperative exposure. The paired point matching using six characteristic anatomic landmarks on the posterior bony surfaces and the surface matching were performed as described above.

The entry point of the optimal axis of pedicle screw insertion (trajectory) was identified with the space pointer and prepared by drilling a shallow hole with a short $2 \mathrm{~mm}$ hand drill. The pilot hole was prepared under image guidance, along the chosen trajectory, with the $3.6 \mathrm{~mm}$ diameter drill bit fitted in the pneumatic hand drill. No additional visualization or orientation aid was used. Both pedicles then had a $3.5 \mathrm{~mm}$ diameter steel cylinder inserted in the resulting holes to increase their visibiiity in the $X$-rays.

The pedicles were then sectioned perpendicular to the screw axes with a precision band saw $(1.5 .-2.5 \mathrm{~mm}$ section thickness). All histological sections were $X$ rayed and classified. To simulate a standard pedicle screw circles with a $6 \mathrm{~mm}$ diameter were concentrically projected onto the image of the metal cylinder in each histological section.

77 histological sections were analyzed. A screw position centered in the pedicle and either not touching or concentrically engaging the cortical wall was considered as ideal. This was achieved in 70 cases. A slight deviation from the ideal axis with an engagement of the pedicular cortex up to one half of its thickness was observed in 7 cases. No cases were found with perforation of the pedicular walls.

Conclusion. The proposed system fulfills the previously defined demands: It complements established surgical techniques and associated tool sets effectively keeping the surgeon "in the loop". It provides sufficient in vitro accuracy and real-time visualization of surgical tools. Since there is no need for intra-operative image intensifying and the process of identification of landmarks is guided the total time of the surgical intervention should not be affected. We are in the process of establishing the CAS system in a specific operating theatre to gain further experience not only in transpedicular screw insertion, but also in further orthopaedic applications, e.g. periacetabular osteotomy, implantation of acetabular prosthetic components, cruciate ligament surgery, etc. In our opinion, the proposed system of CAS has the potential to become a useful tool in daily orthopaedic surgery. 